\title{
Hypothesis testing for the parameters of log-logistic regression model with left-truncated and right-censored survival data
}

\begin{abstract}
ABSTRCT
Left-truncation and right-censoring (LTRC) arise naturally in lifetime data. Data may be lefttruncated due to a limitation in the study design. Failure of a unit is observed only if it fails after a certain period. Usually, the units under study may not be followed until all of them have failed but the study has to be stopped at a certain time. This introduces the right censoring into the survival data. Log-logistic model is extended to accommodate the left-truncated and rightcensored survival data. The bias, standard error (SE), and root mean square error (RMSE) of the parameter estimates are computed to evaluate the performance of the model at different sample sizes, censoring proportion (CP), and truncation level (TL). The results show that the SE of the parameter estimates increase as the truncation level (TL) and censoring proportion (CP) increase. Having low and high TL (5\% and 15\%) in the data, the graphs clearly show that the empirical power of both tests increases with the increase of TL for parameter and. The SE and RMSE also decrease as the sample size increases. Following that, power analysis is conducted via simulation to compare the performance of hypothesis tests based on the Wald and Likelihood Ratio (LR) for the parameters. The results clearly indicate that the Wald performs slightly better than the LR when dealing with the proposed model.
\end{abstract}

Keyword: Log-logistic regression model; Left-truncated and right-censored; Wald; Likelihood ratio; Empirical power 